\title{
Fully-automated liberalism? Blockchain technology and international cooperation in an anarchic world
}

\author{
Bernhard Reinsberg ${ }^{1,2 *}$ (D) \\ ${ }^{1}$ School of Social and Political Sciences, University of Glasgow, 40 Bute Gardens, Glasgow G12 8RT, UK \\ and ${ }^{2}$ Centre for Business Research, University of Cambridge, Cambridge, UK \\ ${ }^{\star}$ Corresponding author. E-mail: bernhard.reinsberg@glasgow.ac.uk
}

(Received 5 July 2019; revised 27 January 2020; accepted 11 May 2020; first published online 5 June 2020)

\begin{abstract}
A recent wave of scholarship attests that the liberal world order is under threat. Although there is disagreement about the underlying reasons for this diagnosis, there are few attempts to further our understanding of how the liberal order can be reinvigorated. This paper probes the potential of blockchain technology to promote international cooperation. Blockchain technology is a data structure that enables global governance stakeholders to establish decentralized governance systems which provide high-powered incentives for enhanced cooperation. By outlining the contours of a blockchain-based global governance system for climate policy, the paper illustrates that blockchain technology holds theoretical promise to foster cooperation in three ways: leveraging new sources of information through blockchain-based prediction markets; allaying coordinating problems through reducing the cost of transactions for side payments; and allowing states and other global governance actors to make more credible commitments given guaranteed execution of blockchain-enabled smart contracts. By empowering local knowledge holders and non-state actors that traditionally lacked the means to coordinate efforts to influence global politics, blockchain technology also promises to advance an international order based on liberal values. In actuality, however, emerging blockchain-based global governance systems will fall short of the libertarian ideal of 'fully-automated liberalism' as their design and operation will remain under the shadow of power.
\end{abstract}

Keywords: blockchain; smart contracts; decentralized governance; climate change; Paris Agreement; liberalism JEL Classification: F30; O19; O30

Liberalism is a theory of international politics that emphasizes international institutions and global society as facilitators of peace and prosperity. ${ }^{1}$ Although the liberal world order is currently under stress, ${ }^{2}$ there is little hope that this state of

\footnotetext{
${ }^{1}$ Ikenberry 2001, Johnson and Heiss 2018, Keohane 1984.

${ }^{2}$ Andersen 2019, Hooghe et al. 2018, Ikenberry 2018.

(c) The Author(s), 2020. Published by Cambridge University Press. This is an Open Access article, distributed under the terms of the Creative Commons Attribution licence (http://creativecommons.org/licenses/by/4.0/), which permits unrestricted re-use, distribution, and reproduction in any medium, provided the original work is properly cited.
} 
nature will change any time soon. International institutions - given the rise of emerging powers - are no longer underpinned by a single hegemon to which all other actors defer. Therefore, they are ever less able to act as focal points and thus fail to reduce the uncertainty that prevents deeper cooperation. Ironically, a return to hegemony would not promote liberal values. Hegemony tends to undermine itself as it creates rules that benefit the hegemon itself and that cannot prevent the hegemon from breaking the rules. ${ }^{3}$ A key challenge in liberal thought therefore is how to promote stability without the need for hegemony.

This paper probes whether recent technological advances - specifically blockchain technology - can promote institutionalized cooperation and whether the creation of blockchain-based governance systems would be normatively desirable from a liberal perspective. A blockchain is a database whose entries are cryptographically linked and which is distributed across participants of a peer-to-peer network. These design principles make a blockchain an incorruptible record of information that network participants can trust even without the existence of a central authority. Although blockchain is merely a data structure, it can be deployed to create so-called 'smart contracts'. These contracts act autonomously in accordance with pre-coded rules, thereby exerting governance effects and potentially changing how societies interact in fundamental ways. ${ }^{4}$

Using the example of climate change as an issue area, I sketch out the contours of a 'Decentralized Autonomous Organization' (DAO) - a virtual entity governed by a set of interrelated smart contracts - that represents a utopian vision of how climate governance would be implemented in a blockchain world. This climate DAO would not only implement key provisions of the Paris Agreement but also be adaptable to changing collective preferences over how global climate policy should be governed.

This example provides the background for subsequent theoretical analysis, which shows that blockchain technology can promote international cooperation, under relatively benign assumptions. In essence, I show that smart contracts provide the building blocks for tapping unmet potential for cooperation in the following three ways: first, they help leverage the wisdom of crowds to validate events in the real world by facilitating prediction markets; second, they help allay distributional problems by automating issue linkage and side payments and realizing transaction cost savings; third, they allow stakeholders to make more credible commitments given the potential for elimination of uncertainty around contract enforcement.

The key theoretical contribution of my paper is to demonstrate how blockchain technology operationalizes the liberal-institutionalist promise of promoting cooperation in an anarchic world. ${ }^{5}$ The hypothetical governance system that I describe here involves global governance actors - states, international organizations, and non-state actors - interacting through interlocking sets of smart contracts. This system promises to promote stability without the need for hegemony, relying on a decentralized system and self-governing contracts that do not require the upfront investment into centralized institutions typically undertaken by a hegemon. Once

\footnotetext{
${ }^{3}$ Keohane 1984, Jervis 1999, Stone 2011.

${ }^{4}$ Wright and De Filippi 2015.

${ }^{5}$ Jervis 1999, Keohane 1984, Stein 2009.
} 
states submit themselves under such a system, they could reap the benefits of reduced uncertainty around enforcement, provided that they staked resources to underpin their commitments. States would also be able to reduce transaction costs by tapping into new sources of trusted information and settling their contractual obligations more efficiently. By leveraging new sources of information and resources from transnational actors with a staked interest in policy change, blockchain technology could promote cooperation even where opportunities for mutual gains have traditionally not been considered to exist. ${ }^{6}$

Obviously, the ability of states to realize the cooperation benefits of blockchain technology depends on their general willingness to cooperate in pursuit of mutual gains, as presumed by neoliberal institutionalism. Although the effectiveness of blockchain-based global governance hinges upon voluntary participation of state actors, a fundamental challenge is how to incentivize the participation of (especially) powerful states, which face the highest cost of submitting themselves under hard contractual obligations. In line with realist thought, it must be expected that such states would either opt out of blockchain-based governance, or would only make shallow commitments. ${ }^{7}$

And yet, the real benefit of blockchain-based global governance is that buy-in from states is not necessary to promote cooperation among non-state actors toward achieving common objectives. This is because blockchain technology indeed allows any kind of stakeholder to make credible commitments, which generates stable expectations about their future behavior which in turn provides incentives for longterm investments in line with such commitments. I therefore argue that blockchain technology has potential to instantiate decentralized governance platforms that implement liberal ideals of a 'fully-automated liberalism' - whereby individual actors and the autonomous contracts that these actors create would work to achieve common objectives. By affording individual actors the possibility to securely transact with each other without the need for central authorities, blockchain technology tends to empower traditionally underprivileged actors. However, the definition of common objectives does not happen in a vacuum but would reflect the pre-existing power distribution. Blockchain technology itself thus has limited potential to fundamentally alter how power is distributed.

My paper makes three additional contributions. The first is to recognize the institution-like properties of blockchain technology. In particular, smart contracts under the aforementioned DAO - by removing uncertainty around enforcement for specific types of conditions - generate fundamentally different expectations about behavior than traditional forms of global governance, thereby meeting the definition of international institutions as sets of interconnected rules that shape actor behavior. ${ }^{8}$ Touching upon issues of contract enforcement, there is substantive literature on international courts. ${ }^{9}$ By examining smart contracts, my paper provides a complementary approach to enforcement and discusses conditions under which they can solve enforcement problems.

\footnotetext{
${ }^{6}$ Krasner 1991.

${ }^{7}$ Downs et al. 1996.

${ }^{8}$ Keohane 1984, Keohane and Martin 1995, North 1990.

${ }^{9}$ Abbott and Snidal 2000, Simmons and Danner 2010, Voeten 2014.
} 
In addition, I contribute to a small literature examining the relationship between cooperation dilemmas, international institutions, and technologies. ${ }^{10}$ Specifically, scholarship within Science and Technology Studies has examined how big data changes the nature of International Relations (IR) and related notions of power, ${ }^{11}$ further highlighting that the design of technologies such as the internet is itself subject to global power struggles and mediated by socio-political norms. ${ }^{12}$ Some domestically-focused public policy research examined how technological innovations may disrupt organizational routines, ${ }^{13}$ whereas others emphasized how the growing availability of low-cost information technology facilitated efficiency-driven outsourcing of government services, raising concerns about democratic legitimacy and data protection. ${ }^{14}$ Although these studies have looked at a range of technologies, no study has focused on blockchain technology, let alone attempted to examine whether and how it could help address cooperation dilemmas at the international level.

Finally, my paper is related to scholarly debates on global order. ${ }^{15}$ Here my contribution is to highlight that blockchain technology, specifically through algorithmic governance, ${ }^{16}$ renders the assumption of anarchy in the international system increasingly irrelevant. As McConaughey, Musgrave and Nexon (2018) noted in this journal already, world politics is best understood as a set of 'nested governance assemblages', even though some relations between some actors may remain anarchical. Some blockchains exist and can affect global politics without the possibility of states to censor them, thus establishing governance at levels below the nationstate. In this way, it promises to empower weaker parties - less powerful states, nongovernment actors, and individuals - providing a tool to make global governance more transparent, if not accessible. Yet, although blockchain technology is considered by its libertarian proponents as an entry point for challenging existing hierarchies, the most-likely context in which it is used in global governance will not radically reconfigure existing hierarchies.

\section{Blockchain technology \\ What is blockchain technology?}

'Blockchain technology' has become a catch-all term for what basically is a bundle of pre-existing technologies whose combination gives rise to socially powerful emergent properties. First and foremost, blockchain is a data structure - a digital ledger in which information is stored in blocks and linked to previous blocks. ${ }^{17}$ Blocks are linked via cryptography, allowing participants with read-access rights

\footnotetext{
${ }^{10}$ Campbell-Verduyn and Goguen 2018, Reinsberg 2019, Ruggie 1975.

${ }^{11}$ Zwitter 2015.

${ }^{12}$ DeNardis 2014, Jasanoff 2004, Just and Latzer 2017.

${ }^{13}$ Beckert 1999, Bendrath and Mueller 2011, Dacin et al. 2002.

${ }^{14}$ Heeks and Bailur 2007, Zysman and Newman 2006.

${ }^{15}$ Krisch 2017, Mattern and Zarakol 2016, Musgrave and Nexon 2018.

${ }^{16}$ Just and Latzer 2017.

${ }^{17}$ My definition of blockchain technology is deliberately broad and includes what some prefer to call 'distributed ledger technology' (DLT), which often entails limited uses of the technology for the purpose of enhancing transparency, typically under the control of centralized actors (Hütten and Thiemann 2018).
} 
to verify that a transaction occurred whereas preserving data privacy. Blocks are transmitted to a distributed network of computers, thereby building resilience against attacks. To ensure that the data are synchronized, every blockchain needs a consensus mechanism. If a network participant wants to initiate a new transaction creating a new block in the blockchain - all network participants must first accept its validity. As the integrity of a participant cannot be readily assumed, the consensus mechanism holds key to the sanctity of the data.

It is important to realize that there is no single type of blockchain. Different blockchains vary with respect to rules of access and scope of distribution. In so-called 'permissionless blockchains', everyone can join the blockchain network and write consensus data. Such highly decentralized systems - due to their need for consensus, layers of encryption, and redundancy - reduce the speed with which transactions can be settled. ${ }^{18}$ In so-called 'permissioned blockchains', the ability to manipulate the blockchain is restricted to pre-approved participants who need off-network authentication and permission to write. ${ }^{19}$ Permissions can be issued by a central authority; alternatively, consortium systems rely on the collective decision of participants, without involving a central authority. In a permissioned network, the identity of every participant is known. Permissioned blockchain networks do not abrogate the requirement that every node on the network perform all of the computation for the entire network, but they break this computation into particular segments and thereby increase overall performance and reduce transaction costs relative to permissionless blockchains. ${ }^{20}$ An example is a consortium of banks that use a private blockchain to settle derivatives.

In addition, there are differences as to who is authorized to read information from a blockchain. In public blockchains, everyone with an internet-connect device and the related software can read-access the blockchain, whereas private blockchains require authentication for this purpose. ${ }^{21}$ Obviously, there is a correlation in the relationship between write-restriction and read-restriction, although the two design dimensions are analytically separate. Their combination allows for a variety of blockchain designs that can be adapted to the specific nature of the given problems.

For example, Bitcoin - the first and best-known application of a blockchain uses a public-permissionless blockchain, conceived by its founder as a decentralized alternative to fiat money backed by central banks. ${ }^{22}$ This blockchain design reflects its purpose of enabling direct instantaneous transfer of value among participants in a fully decentralized manner - without the need for participants to trust each other and without a need for a central authority to settle transactions. ${ }^{23}$ The downside of a completely open consensus mechanism is that fraud - in particular doublespending of bitcoins - must be made sufficiently unattractive. Bitcoin solves this by requiring 'proof of work' - the costly computation of a complex cryptographic problem - which has been criticized for its high-energy consumption. ${ }^{24}$

\footnotetext{
${ }^{18}$ Pisa 2018.

${ }^{19}$ Valkenburgh 2016.

${ }^{20}$ Baliga 2017.

${ }^{21}$ Valkenburgh 2016.

${ }^{22}$ Nakamoto 2008.

${ }^{23}$ Sklaroff 2017.

${ }^{24}$ Truby 2018.
} 
Public-permissioned blockchains, which involve an element of authentication, have already been deployed for digital identity services, land registries, supply-chain management, decentralized file storage, and crowdfunding climate finance. ${ }^{25}$ These applications are often built upon Ethereum ${ }^{26}$ - a general-purpose blockchain that can settle any kind of digital transactions, not just cryptocurrency transfers. Aside from the proof-of-work consensus mechanism, Ethereum supports 'proof of stake', whereby a participant gets chosen to validate a proposed block with probability proportional to its staked deposit. If a proposed block does not get included in the blockchain, a validator loses its staked deposit. ${ }^{27}$ Yet another consensus mechanism - used by the World Bank in its recent sale of a blockchain bond - is 'proof of authority', which lets trusted participants (such as the World Bank) manipulate the blockchain. ${ }^{28}$

\section{Blockchain-enabled functionalities}

A key blockchain-enabled functionality that merits particular attention - given its potential use for global governance - is the 'smart contract', ${ }^{29}$ which facilitates, validates, and records transactions and agreements between multiple parties. ${ }^{30}$ Smart contracts allow many types of contractual clauses to be made self-executing, selfenforcing, or both. ${ }^{31}$ An example is a so-called 'multi-signature escrow', whereby a contract executes when a specified quorum of participants have endorsed it through their digital signatures. ${ }^{32}$ In IR, the equivalent of an escrow would be an arbitrator whose consent is required for a bilateral dispute to be settled if the two states disagree. If the contract specifies that a transaction needs to be digitally signed by at least two participants, the arbitrator becomes the pivotal player if only one of two parties has provided its signature. ${ }^{33}$ Smart contracts hold most promise to reduce transaction costs for routine activities, for example to settle market transactions, where a buyer could use a smart contract to automatically release payment to a supplier of a good once the good has arrived at a specified location. ${ }^{34}$

Smart contracts provide the building blocks for so-called decentralized applications (dApps), which essentially combine several smart contracts. ${ }^{35}$ An even more sophisticated application - 'decentralized autonomous organizations' (DAOs) -

\footnotetext{
${ }^{25}$ Swan 2015, 22.

${ }^{26}$ Buterin 2014.

${ }^{27}$ Baliga 2017.

${ }^{28}$ https://t.co/tieQoQ9uLe (accessed 27 October 2018).

${ }^{29}$ Szabo 1994.

${ }^{30}$ Rocamora and Amellina 2018, 14.

${ }^{31}$ There are many blockchain platforms which can implement smart contracts. Ethereum - a generalized blockchain - allows for such applications through the Ethereum virtual machine (EVM) - a computer running on top of the Ethereum blockchain that can both encode programs and execute them (Swan 2015, 21). In principle, similar functionality is provided by Hyperledger, an umbrella project of open-source blockchains started by the Linux Foundation and supported by industry (Tapscott and Tapscott 2017).

${ }^{32}$ Diedrich 2016.

${ }^{33}$ Tapscott and Tapscott 2016, 104.

${ }^{34}$ Krishna et al. 2017.

${ }^{35}$ DApps can be built on the EVM. By running on a blockchain (such as Ethereum) they import its desirable properties such as pseudonymity, censor-proofness, auditability, and transparency (Swan 2015).
} 
link several smart contracts together to form a self-governing system. ${ }^{36}$ A DAO is a virtual entity that has a certain set of members which have the right to modify its code and spend its funds. ${ }^{37}$ For example, DAO members could decide to change quorums required for certain decisions. ${ }^{38}$ DAOs mimic existing collective entities such as international organizations, but use smart contracts for enforcement. ${ }^{39}$ DAOs have come under criticism after the infamous 2016 DAO hack, in which an attacker exploited a loophole in the coding protocol to siphon off DAO funds. ${ }^{40}$ In the DAO hack, no rule was agreed ex ante how to deal with attacks, which led to an ad-hoc human decision to fork the blockchain. ${ }^{41}$ In any DAO, though, parties to smart contracts could agree on how hacks can be addressed, even though these will vary in style.

A common challenge for all smart contracts is that unless information is readily available on the blockchain, as for example for cryptocurrency transfers, they depend on external information feeds - so-called 'oracles' - to bring the relevant information on the blockchain. With the rise of the 'Internet of Things' (IoT), tamper-proof smart devices are becoming increasingly important technical oracles. ${ }^{42}$ Another kind of oracle, facilitated by smart contracts, are 'blockchain-based prediction markets', which aggregate information about the occurrence of events in a non-manipulable way. Although prediction markets have existed long before the arrival of blockchain, they were malfunctioning because no central authority could commit to not exploit sensitive information for its own gain. As a result, reported information was not trustworthy. Blockchain technology, however, supports the proper functioning of prediction markets that leverage the 'wisdom of crowds'. The decentralized nature of blockchain-based prediction markets implies that no one effectively controls information whereas information can be shared securely to validate certain events. An internal reputation system - drawn from game-theoretic approaches of mechanism design - incentivizes truthful reporting. ${ }^{44}$

\footnotetext{
${ }^{36}$ Buerger 2016, Diedrich 2016, Swan 2015.

${ }^{37}$ The simplest DAO design involves a piece of self-modifying code that changes if a qualified majority of members agree on a change. Although code is theoretically immutable, it can be de-facto muted by having chunks of the code in separate contracts, and having the address of which contracts to call stored in the modifiable storage.

${ }^{38} \mathrm{DAOs}$ would be governed by three transaction types, distinguished by the data provided in the transaction: proposal transactions, transactions to vote for a proposal, and transactions to finalize a transaction provided enough votes were made (Buerger 2016).

${ }^{39}$ There also is a literature on how DAOs can maintain the social contract within nation-states (Atzori 2015). This is the application of my argument that blockchains can deliver solutions to collective action problems without a hegemon to the national level.

${ }^{40}$ DuPont 2018.

${ }^{41}$ For some, this reliance on a 'hard fork' as a form of meta-governance represents a deliberate governance decision, showing that the DAO was in fact viable (Zwitter and Hazenberg 2020).

${ }^{42}$ In practice, smart devices may be tamper-proof only under certain conditions. Consider a solar sensor that could be manipulated by shining an UV lamp on it. And yet, decentralized oracle networks such as the ones afforded by ChainLink are a first good attempt to address the 'oracle problem' (Orcutt 2018).

${ }^{43} \mathrm{https} / / /$ medium.com/@ConsenSys/why-how-decentralized-prediction-markets-will-change-just-abouteverything-15ff02c98f7c (accessed 23 August 2018).

${ }^{44}$ In the case of Augur, described below, reporters receive half of the fees in the system multiplied by the token shares they own. Because participants of the prediction market have an incentive to not undermine the value of the token, they have incentives to truthfully report.
} 
Prediction markets are accurate because market participants stake real money on their predictions, whereas participants report valuable information given guaranteed payments through smart contracts. ${ }^{45}$ Smart contracts thus not only depend on prediction markets for their functionality but also ensure their viability.

\section{Blockchain-based global governance}

The central idea of this paper is that blockchain technology - by facilitating transactions between actors and the autonomous contract agents that they create - is a decentralized mechanism for global governance, which can be defined as 'systems of rule at all levels of human activity - from the family to the international organization - in which the pursuit of goals through the exercise of control has transnational repercussions. ${ }^{46}$

Although the ability to govern has traditionally been confined to human beings, the rise of information and communication technologies, specifically computer algorithms, has come to challenge this view. Scholars have coined the term 'algorithmic governance' to refer to computer programs making authoritative decisions that construct reality and social order, based on predefined code and often using big data as input. ${ }^{47}$ Blockchain governance - as a subset of algorithmic governance refers to those algorithms that run simultaneously on a distributed network of computers. Algorithmic governance can extend across national boundaries, for instance through algorithmic selection on the internet, or indeed through transnational relations on blockchain platforms.

Competing perspectives exist to understand the implications of blockchain governance for global society. What unites them is their recognition that blockchain technology - by giving rise to autonomous agents who follow encoded scripts - exert a governing effect on social behavior. They can do so at least by altering the relative attractiveness of different behavioral choices, if not by altering preferences toward cooperation themselves, given that actors come to understand that 'code is law'. ${ }^{48}$

Blockchain governance still is not an entirely accurate label for my subsequent analysis. It may refer to governance by the blockchain (i.e. cooperation between stakeholders through blockchain-based systems) and governance of the blockchain (i.e. how stakeholders take decisions about the rules by which the blockchain operates, the so-called 'protocol'). To clarify that my main interest in this paper is in the ways in which blockchain technology can be used to further cooperation at the global level rather than the principles by which blockchains themselves are governed - I henceforth use the term blockchain-based global governance, not blockchain governance.

\footnotetext{
${ }^{45}$ At least two blockchain-based prediction markets are operational. The first is Augur (https://augur.net/, accessed 22 October 2017) - a decentralized prediction market which runs on a network of servers and cannot be manipulated. All participants report on the outcome using a consensus-based mechanism. Safe payment is ensured via smart contracts. A second is Gnosis (https://gnosis.io/, accessed 21 January 2018), which operates 'prediction market platforms allow[ing] anyone to utilize customized forecasting applications [using] an entirely new asset class: conditional tokens, which make event outcomes tradeable and are also a powerful tool for information discovery'.

${ }^{46}$ Rosenau 1995, 1.

${ }^{47}$ Just and Latzer 2017.

${ }^{48}$ Lessig 1999.
} 


\section{Blockchain-based global governance for climate policy Why climate governance?}

I demonstrate the implementation of blockchain-based global governance for the issue area of climate change. First of all, the combat against climate change is fraught with a diversity of complex cooperation problems, which allow for probing the different ways in which blockchain technology might help address such problems. In the domain of mitigation, a key challenge is to prevent states from freeriding on emission reductions of other states. In the domain of adaptation, states face an international distribution problem over how to spread the costs of financing the adaptation to the inevitable consequences of global warming. Both domains are not independent - as less ambitious collective mitigation efforts imply a greater need for more adaptation efforts specifically in the most vulnerable countries which further complicates the bargaining process. Finally, the global fight against climate change also entails a distribution problem at the domestic level. Domestic bargains determine the viability of international bargains on climate policy, but in many countries the prospect for domestic bargains is bleak due to powerful lobbies with vested interests in the carbon economy.

At the same time, the fight against climate change has brought to the fore an unparalleled diversity of actors with the capacity to take globally coordinated action, regardless of concurrent efforts led by national governments. Around the globe, substate actors - in particular cities - coordinate their efforts to hasten green urban transformations whereas at the same time lobbying their national governments to embrace de-carbonization. ${ }^{49}$ Cities have institutionalized their climate-related efforts through transgovernmental networks such as the C40 Cities Climate Leadership Group, International Council for Local Environmental Initiatives (ICLEI), and the Coalition for Urban Transitions, which recently published an influential study illustrating how the development of carbon-neutral cities would help solve the climate crisis. ${ }^{50}$ There even is a proposal for a world parliament of cities that deliberates and undertakes to do whatever they are willing to do voluntarily, but under traditional governance this entity would remain under the purview of states. ${ }^{51}$

The climate regime is one that is rife with private-led initiatives using blockchain technology, upon which states could draw to operationalize their climate-related commitments. Besides global emissions trading markets, ${ }^{52}$ an important blockchain-based application is climate-related crowdfunding. WeiFund provides an example of an open crowdfunding platform implemented through smart contracts on the Ethereum blockchain. ${ }^{53}$ Other projects seek to find innovative ways to mitigate the carbon footprint of blockchain technology itself, for instance by using transaction fees in the network to buy real-world carbon credits. ${ }^{54}$ Finally,

\footnotetext{
${ }^{49}$ Barber 2013, Baumann 2018, Bulkeley et al. 2014.

${ }^{50}$ https://urbantransitions.global/en/publication/climate-emergency-urban-opportunity/ (accessed 1 January 2020).

${ }^{51}$ Barber 2013.

${ }^{52} \mathrm{Fu}$ et al. 2018.

${ }^{53}$ Aganaba-Jeanty et al. 2017.

${ }^{54}$ See http://carbongrid.io (accessed 10 May 2018). Also, www.cleancoins.io (accessed 10 May 2018) is a project to assess the carbon footprint of blockchains and scout solutions to reduce their emissions.
} 
blockchain technology is already being used for peer-to-peer energy trading. For example, SolarCoin is a community-based solar electricity reward program. ${ }^{55}$ Any solar installation can register with the network and receive SolarCoins for a verified amount of solar electricity produced. Users can spent coins for solar energy within the network or exchange them for fiat currencies. ${ }^{56}$

The blossoming ecosystem of climate initiatives also reminds us that climate change - albeit plagued by cooperation problems among states - is the product of the sum of individual decisions and addressing it would likely require incentive systems that foremost change individual behavior. In the following, I highlight the key provisions of the Paris Agreement - as the most recent accord that will govern climate-related cooperation in the foreseeable future - and describe how bringing them on the blockchain would create the contours of an emerging 'climate DAO' that would help stakeholders address underlying cooperation problems.

\section{From the Paris Agreement to the climate DAO}

The Paris Agreement - the successor regime of the Kyoto Protocol - marks a momentous turn in the governance of climate change. Unlike the Kyoto Protocol, it does not require states to make binding commitments, but instead takes a bottom-up approach in which states set their own targets - so-called Nationally Determined Contributions (NDCs) - and communicate them internationally, reviewed by an expert panel. ${ }^{57}$ Hopes are that reputational concerns and network effects would entice states to commit to more ambitious NDCs. ${ }^{58}$

Bringing the Paris Agreement on the blockchain would entail that member states first create a permissioned blockchain. Write-access would be restricted to preidentified stakeholders such as states themselves and entrusted third parties - international organizations, non-governmental organizations, and expert bodies. States may decide to make some information visible to everyone outside the system. In particular, publicly recording the NDCs would meet the transparency requirements of the Paris Agreement (Article 4). Once the data are recorded on the blockchain, it is extremely hard to forge by anyone. This implies reduced administrative costs by obviating the need for the UNFCCC secretariat to keep a central record of such NDCs.

With the ability to purchase carbon credits in other jurisdictions comes the need for 'robust accounting' that seeks to avoid double-counting of emission reductions (Article 6). The transparency-related functionalities of blockchain technology would make it a cost-effective solution to this problem, circumventing the need for a central organization to settle transactions of carbon credits. ${ }^{59}$ In fact, the Pacific Alliance nations are planning to create a blockchain-based monitoring system for emissions. ${ }^{60}$

\footnotetext{
${ }^{55}$ www.solarcoin.org (accessed 10 May 2018). A similar project is LO3 energy (Aganaba-Jeanty et al., 2017).

${ }^{56}$ Wigley and Cary 2017, 28.

${ }^{57}$ Hale 2016.

${ }^{58}$ Falkner 2016.

${ }^{59}$ UNFCCC 2017.

${ }^{60}$ Wigley and Cary 2017, 28.
} 
For other provisions of the Paris Agreement, a more extended use of blockchain technology is necessary. In particular, states would need to intertwine their blockchain with the ecosystem of permissionless blockchains in the climate sector. Technically, this can be implemented through 'heterogeneous multi-chain frameworks', ${ }^{61}$ which enable different kinds of consensus systems in a 'federation' to have trust-free access to each other. A common natural token, which might be called 'greencoin' and which would be created on top of a permissionless blockchain (such as Ethereum), would ensure that different blockchains would be interoperable. For example, states could use tokens to hire other stakeholders to perform certain tasks.

One additional task such a blockchain-based governance system could address is to facilitate 'results-based payments' for 'activities relating to reducing emissions from deforestation and forest degradation' (Article 5). To see how this would work in practice, imagine that anyone in a permissionless network connected to the system can earn greencoins by planting trees ${ }^{62}$ (equivalent to 'mining' in the Bitcoin system); however, to ensure that greencoins have actual value, states could stake real money on them - through a smart contract residing on the permissioned blockchain that takes a monetary deposit from each state. If states fail to comply with their emission reduction targets, their deposit will be taken and redistributed as greencoins to individuals that have planted trees. Instead of planting trees, individuals could also buy up greencoins to support climate action. By increasing the exchange value of greencoins, these individuals would provide incentives for more rapid tree-planting. Obviously, tree-planting is just one example of climate-related activities; states could agree on a list of potential measures along with ways of verification and the incentive system underlying effective decentralized verification.

Given the elevated role of climate-related information (Article 13), there is a need for robust systems that produce information that can be trusted. Blockchain technology - particularly smart contracts - facilitate information provision by IoT devices and prediction markets, hence contributing to the verification of emission reductions and adaptation measures at the local level. Smart contracts also offer a way to securely reward local information providers with greencoins, thus meeting the Paris Agreement principle of 'drawing on [the] knowledge of indigenous peoples and local knowledge systems' (Article 7).

The benefit of a common token in the blockchain system is to allow for a panoply of measures to be rewarded, whereas further promoting climate ambition (Article 6), even at the highest political levels. For example, consider the decision by US president Donald Trump to withdraw from the Paris Agreement, motivated with the expected job losses for carbon-intensive industry workers. Major transnational corporations - including Apple, Google, and Walmart - and even fossil fuel firms such as ExxonMobil, BP, Shell, and other firms outside the USA criticized the decision and reaffirmed their support for the Paris Agreement. ${ }^{63}$ A series

\footnotetext{
${ }^{61}$ Wood 2017.

${ }^{62}$ In fact, research shows that tree planting has enormous potential to capture emissions (https://www. theguardian.com/environment/2019/jul/04/planting-billions-trees-best-tackle-climate-crisis-scientists-canopy-emissions, accessed 5 July 2019) although other measures could be eligible.

${ }^{63}$ The Guardian, 'Donald Trump confirms US will quit Paris climate agreement', accessible via https:// www.theguardian.com/environment/2017/jun/01/donald-trump-confirms-us-will-quit-paris-climate-deal (23 May 2018).
} 
of smart contracts could have facilitated the compensation of affected workers using payments from transnational corporations benefiting from the low-carbon transition. Under the existing system, such a complex transnational arrangement would have involved high transaction costs without giving stakeholders the certainty that all transactions would settle. ${ }^{64}$

Last but not least, the above blockchain-based system can be further developed into a climate DAO that would allow participants to alter procedural rules. To be sure, procedural clauses such as the requirement that 55 states representing $55 \%$ of global emissions must have ratified the Paris Agreement before it enters into force (Article 21) are easily translated into the language of smart contracts. Clauses such as these could be changed by self-modifying pieces of code. Any country could propose to alter these rules, by releasing a transaction to vote for a proposal. States vote on proposals by spending their tokens on a given proposal. In other words, a token would operationalize a voting mechanism for on-chain governance, such that states would have incentives to invest more tokens for proposals about which they care more.

The here-described climate DAO is a self-governed organization controlled by an incorruptible (yet collectively adjustable) set of rules, implemented as a set of smart contracts. Some encode contractual relationships between states, international organizations, and non-state actors. Once established by stakeholders, smart contracts interact with each other and with other stakeholders according to their predefined code, without the need for human triggers. ${ }^{65}$ For example, some contracts may pay smart devices for feeding critical information into the system. In the same way, smart contracts can be fully self-executing, thereby punishing state behavior that violates a previously made commitment. International cooperation would thus come about through the interaction of (in many cases fully autonomous) smart contracts.

\section{Theoretical implications}

Having laid out the principal design of blockchain-based global governance with an illustration of a climate DAO, I now discuss the implications of such a governance system for international cooperation, normative issues, and global order.

\section{Cooperation}

Blockchain technology promises to promote international cooperation in three ways. First, it could address information problems by leveraging distributed consensus to generate reliable information. Second, it would offer a secure and efficient way of making side payments as part of agreements, hence allaying distribution problems. Third, blockchain technology could enhance the credibility of state

\footnotetext{
${ }^{64}$ Staking of deposits is another possibility to tie the hands of future governments to exit from the Paris Agreement. If then-US president Barack Obama had staked money on the agreement, the effect of the subsequent US exit could have been fully neutralized as the staked deposit would have been channeled to the Green Climate Fund to support adaptation projects.

${ }^{65}$ De Filippi and Hassan 2016.
} 
commitments by allowing for guaranteed execution of intergovernmental contracts under certain conditions. I discuss each of these promises in more detail below.

Leveraging new information: Blockchain technology may be deployed to alleviate information problems, given the way it represents data and the difficulty to tamper with the data. In general, blockchain technology is particularly useful where participants need to access, verify, send, or store information securely, but where a trusted central authority for that purpose is not readily available. ${ }^{66}$ It also holds significant promise wherever verification that an event happened is necessary but prohibitively costly.

Although information that has once been recorded on the blockchain is resistant to manipulation, a remaining challenge is how to ensure the accuracy of such information in the first place. The usefulness of blockchain technology in this regard depends on the type of information to be stored. Efficiency gains are largest for 'on-chain events' - the ones that occur within the blockchain system, such as transfer of digital currency. The more challenging case refers to 'off-chain events' - those that occur in the real world but are not yet represented on the blockchain. To verify offchain events, states can use oracles. For instance, satellite imagery is a technological oracle that can verify preservation of rain forest areas that countries might have promised to undertake. A blockchain-based prediction market is another type of oracle, allowing participants to ascertain the probability that a specified event happened. As the international system is characterized by a paucity of information that is often untrustworthy, states which have the most interest in accurate information will act as market makers and provide liquidity to initiate a prediction market.

One might contend that there are enough trusted parties who facilitate state cooperation by serving as mediators, validators, and knowledge producers. ${ }^{67}$ However, stakeholders may not want to delegate authority to such parties, as those may abuse this power to advance their own agendas. For instance, as noted by principal-agent literature, international organizations, if endowed with too much delegated authority, can overstep their mandates and be prone to uncontrolled budget growth. ${ }^{68}$ In such contexts, oracles such as IoT devices and decentralized prediction markets provide an alternative source of trusted information.

And yet, for a range of information types, there is a continued need for trusted third parties, such as international organizations, non-governmental organizations, and expert bodies. ${ }^{69}$ Traditional third parties may be superior to decentralized information providers specifically for highly technical issues, such as monetary policy-making, where it is unlikely that public prediction markets will have many reporters because individuals lack the expertise to make informed bets. In this case, a technocratic international organization may be the better choice. ${ }^{70}$

\footnotetext{
${ }^{66}$ Wigley and Carey 2017.

${ }^{67}$ Abbott and Snidal 1998, Dietrich and Murdie 2017, Lundgren 2016.

${ }^{68}$ Barnett and Finnemore 2004, Nielson and Tierney 2003, Vaubel et al. 2007.

${ }^{69}$ Arruñada 2018, GSMA 2017, Sklaroff 2017.

${ }^{70}$ Another issue is that blockchain-based prediction markets also need to be regulated by central authorities to prevent their use for clandestine purposes - for example, betting on the assassination of a democratic incumbent (Sklaroff 2017).
} 
Allaying distribution problems: If - as some scholars argue - distributional problems are the main impediment to cooperation, the primary role of institutions is not to prevent cheating (because a once-chosen equilibrium is self-enforcing) but to help states choose among multiple equilibria with different distributional implications. ${ }^{71}$ As argued by neoliberal institutionalists, institutions help states to do so by facilitating issue linkage and side payments. ${ }^{72}$

Blockchain technology, particularly smart contracts, can be used to streamline these processes. Each state would encode in a smart contract what it would be willing to surrender in exchange for some benefit that another state would grant. The blockchain as a decentralized ledger of such commitments would then serve as 'market maker' - like a trading computer on the stock market - and identify the Pareto-optimal allocation of benefits. Presumably, the efficiency gains from using blockchains for this purpose are not large but increasing in the extent to which state bargaining follows a standardized protocol, occurs rather frequently, and involves easily traceable or digital assets. Multilateral trade negotiations provide a case in point. These negotiations have long followed an informal procedure by which the most powerful states define the agenda and reach agreement in informal minilateral settings that they seek to extend to the membership at large. As the ambassador of Costa Rica to the WTO stated, trade policy is still being negotiated in a [...] widely inefficient manner" ${ }^{73}$ - as it fails to search the bargaining space for globally optimal deals that make all countries better off. Just like trade negotiations, climate-related negotiations are complex and the unmet potential for Pareto-increasing policy choices is relatively high.

Blockchain technology can also solve gridlock between states by breaking up the domestic bargains that constrain governments to commit to cooperative policies at the international level. ${ }^{74}$ This is particularly relevant when non-state actors - specifically the ones that reside in other jurisdictions - have significant resources available to incentivize pro-cooperation behavior of domestically-constrained governments, as the earlier thought experiment on the US exit from Paris illustrated. By allowing transnational actors to devise smart contracts that promise to pay states for compliance, the resulting blockchain ecosystem would address the staking problem by contributing money from sources other than states.

In sum, blockchain technology has potential to allay distributional problems by offering a way to organize issue linkage and side payments more efficiently through smart contracts. In the context of coordination problems, the rigidity of such contracts is advantageous because once they settle a proposed transaction, states have no incentives to rewind it. In addition, a blockchain-based governance ecosystem promises to shift the Pareto frontier by making available financial resources from non-state actors to incentivize government cooperation.

Making commitments more credible: A final promise of blockchain technology is to allow states to make credible commitments - as states would be able to design contracts whose execution is guaranteed. There are two reasons to believe that states

\footnotetext{
${ }^{71}$ Koremenos et al. 2001, Morrow 1994, Stein 1982.

${ }^{72}$ Keohane 1984.

${ }^{73}$ Troyjo et al. 2017, 60.

${ }^{74}$ Dai 2007.
} 
have interests to do so. First, governments - especially those with limited tenure such as democratically elected ones - have incentives to lock in policy. By adopting institutions from which exit is costly, they can effectively tie the hands of their successors and limit policy reversals - a strategy that is well known in the context of delegating monetary policy to an independent central bank. ${ }^{75}$ Second, the lack of enforcement creates incentives to renege on commitments, which undermines their strength. ${ }^{76}$ Guaranteed enforcement allows states to make more credible commitments to each other because uncertainty around enforcement is removed. As smart contracts can be programed not to enter into force until a quorum of states complies with them, any state wishing to make a stronger commitment does not inevitably expose itself to undue risk of being exploited by other states. ${ }^{77}$

This is a potential advantage over currently existing institutions. Even international organizations - the most centralized entities in world politics with considerable degrees of delegated authority - are far from being direct enforcers of rules but do so indirectly by relying on incentives, persuasion, and learning. ${ }^{78}$ International organizations often serve as commitment devices ex ante, for instance through costly accession procedures, ${ }^{79}$ which are effectively realized costs. International organizations also have limited capacities to enforce state commitments ex post, whereas smart contracts allow for guaranteed punishment when a state violates a previously made commitment.

For smart contracts to effectively enhance the credibility of commitments, states must have staked resources upon them that states would lose if they did not follow through on their promises. For incumbents who contemplate about a commitment, the ability of the smart contact to hold funds securely in custody should reassure them to make a deposit that it might otherwise not be willing to make vis-à-vis a less trustworthy intermediary. ${ }^{80}$ Future incumbents who inherit a stake from their predecessor are effectively constrained by the smart contract because noncompliance will lead to loss of deposit. Full automation of the commitment-compliance process requires that contract conditions can be unambiguously evaluated, which however is the case only for blockchain events (although the rise of IoT devices and prediction markets facilitated by smart contracts enlarges the set of contract conditions that are interpretable). Under such conditions, problems of moral hazard would be eliminated, and costs of verification and enforcement of promises would be reduced.

If enforcement problems and the related lack of credible commitment inhibit intergovernmental cooperation, blockchain technology, and particularly smart contracts, hold promise to promote cooperation. From a merely technical perspective,

\footnotetext{
${ }^{75}$ Boylan 2001, Kern et al. 2019, Tallberg et al. 2016.

${ }^{76}$ Fearon 1995.

${ }^{77}$ Similarly, international law stipulates that a treaty commitment is not binding until a sufficient number of states have ratified it. This provision allays concerns about other states obtaining relative gains from delaying ratification. It can be easily implemented in 'blockchain law' using multi-party escrows (Diedrich 2016).

${ }^{78}$ Dobbin et al. 2007, Kelley and Simmons 2015, Simmons and Elkins 2004.

${ }^{79}$ Martin 2017.

${ }^{80} \mathrm{An}$ early non-digital analog to staking is royal intermarriage. Blockchains extend this notion further by allowing arbitrary resources to be staked on commitments.
} 
the effectiveness of many smart contracts may be limited by their reliance on realworld authorities and their own contractual rigidity that is essential for their efficiency. The first problem may become more manageable in the future, considering the increasing availability of smart devices and blockchain-based prediction markets, which serve as information input devices to the blockchain. A crucial task though would be to increase the resilience of these input devices to manipulation, as they are the key 'infostructures' of the blockchain age. ${ }^{81}$

The second problem is more severe. In fact, there is an inherent tradeoff between efficiency afforded by a smart contract and the flexibility that the language of ambiguous contracts provides. ${ }^{82}$ Smart contracts are fully efficient only if they can execute automatically. To that end, they must be 'complete contracts' ${ }^{83}$ - specifying all possible contingencies upfront. Even if this was feasible, it would be costly for complex issues, thus increasing the cost of ex ante contract design. More importantly, complete contracts may not be collectively desirable. Governance actors may not want to be collectively bound by a formal treaty provision if circumstances change unexpectedly. ${ }^{84}$ Indeed, many international agreements include escape clauses, treaty reservations, non-legally binding provisions, and procedural standards that need further interpretation. This is not accidental but a deliberate design choice by states which desire flexibility, especially when uncertainty about the state of the world is high. ${ }^{85}$

Where blockchain transactions require enforcement in the real world, the blockchain is as ineffective as conventional international law if powerful states renege on their promises in the absence of a world government. And even where execution is possible as in the case of on-chain events, the strength of guaranteed execution might actually turn into a weakness: states anticipating guaranteed punishment for reneging might be unwilling to make ambitious commitments in the first place. This implies that state commitments under smart contracts will likely be trivial, or participation in blockchain-based governance would be limited to states that are willing to comply or that expect to comply with contract obligations. ${ }^{86}$

Cross-cutting considerations: Albeit analytically distinct, the above mechanisms through which blockchain technology can enhance cooperation are interrelated. Smart contracts are key for the effective functioning of oracles, specifically prediction markets. Oracles are sources of new information that would help enlarge the

\footnotetext{
${ }^{81}$ Campbell-Verduyn et al. 2019. Here, a related point would be how to ensure that cryptographic certificates - which are needed to identify parties on a blockchain - would not be stolen. In the event of loss of these keys, there would be no way to differentiate between legitimate and fraudulent transactions. I thank an anonymous reviewer for highlighting this point.

${ }^{82}$ Sklaroff 2017. The issue of how to fix coding bugs in smart contracts is discussed among researchers. Some argue that code needs the law as a fallback option, others maintain that rules for contingencies can be agreed upon by parties upfront.

${ }^{83}$ Hart and Moore 1999.

${ }^{84}$ The inflexibility of smart contracts also poses elevated transactional risks. Smart contracts - even if they contain programing errors - are unstoppable; human discretion to weed out human errors is not part of their protocol (Sklaroff 2017). Although notarial certification could be required for such contracts to execute, this would practically eliminate their efficiency advantages arising from automation.

${ }^{85}$ Abbott and Snidal 2000, Koremenos et al. 2001, Rosendorff and Milner 2001.

${ }^{86}$ Downs et al. 1996, Simmons 2010, von Stein 2005.
} 
set of self-executing smart contracts, thus promoting cooperation by eliminating enforcement uncertainty and realizing gains from reducing the costs of settling contracts. For complex multilateral bargaining situations where states have not identified Pareto-optimal policy outcomes, smart contracts residing on the blockchain could help states to identify mutually preferable bargaining outcomes, thereby reducing transaction costs of policy bargaining and streamlining agreement execution. This suggests that blockchain technology may enhance cooperation by altering state preferences over strategies. ${ }^{87}$

An even more important benefit of blockchain technology is its potential to alter state preferences over outcomes. In some issue areas, there are (resourceful) domestic stakeholders with pro-cooperation preferences who can use blockchain technology to incentivize recalcitrant governments to cooperate, or, failing that, circumvent them altogether. This is important as it shows that - given the technology - there is no longer a need for a hegemon to uphold cooperation. At the same time, cooperation would become truly transnational, thus coming close to the 'open liberal order' that liberal observers have envisioned. ${ }^{88}$ A good example to illustrate this is the recent announcement by Microsoft to invest USD 1 billion over the next decade to become a 'carbon-negative enterprise'. ${ }^{89}$ To help underpin the credibility of this statement, Microsoft could encode this commitment in a smart contract with an appropriate stake. This would make powerful players such as Microsoft truly accountable, whereas providing certainty for other (less powerful) actors to make long-term investments into technologies that would help Microsoft achieve its commitment.

\section{Normative implications: power and legitimacy}

Even if blockchain technology could help promote cooperation, a remaining issue is whether its use would be normatively desirable. Liberalism provides a normative standard against which to judge blockchain-based global governance imaginaries. As a tradition of thought, liberalism emphasizes individual freedom of choice, equality of individuals, and protection against abuse of power. Early liberal thinkers such as Immanuel Kant argued that if it was not for autocratic rulers, human beings and their nation-states would live in harmony with each other. Following from thinkers such as Abbé Saint-Pierre, ${ }^{90}$ Giuseppe Mazzini, ${ }^{91}$ and Woodrow Wilson, ${ }^{92}$ liberalists posit that rulers could bind themselves using international institutions, ${ }^{93}$ which exist to promote economic prosperity and sustain peace and thus make everyone better off.

Blockchain technology seems to be well-placed to promote 'fully-automated liberalism' - a state of nature where participants undertake mutually beneficial

\footnotetext{
${ }^{87}$ This terminology is due to Jervis 1999.

${ }^{88}$ Slaughter 2016.

${ }^{89}$ Microsoft wants to eradicate its carbon footprint by going emissions 'negative' by 2030, https://edition. cnn.com/2020/01/16/tech/microsoft-climate-pledge-2030/index.html (accessed 17 January 2020).

${ }^{90}$ Brown et al. 2002.

${ }^{91}$ Haddock 1999.

${ }^{92}$ Wilson 2006.

${ }^{93}$ Ikenberry 2001.
} 
transactions whereas furthering the collective goals of global society. ${ }^{94}$ Its normative appeal is that all actors, even the most powerful ones, would be constrained by their own promises encoded within smart contracts. The fundamental problem obviously is how to incentivize powerful actors to make such hard commitments. Aside from the technical difficulties to generate self-enforcing contracts discussed earlier, blockchain-based global governance would unlikely be egalitarian but imbued by pre-existing power asymmetries. Similar to other technologies, blockchains are agnostic about the content of the computer code that runs on them. As technologies are always embedded in specific social contexts, blockchain technology, too, will be deployed in ways that reflect existing power structures. Thus, as I will argue, even if a blockchain-based governance system like the climate DAO were to be implemented, it would fall short of liberal ideals as it cannot fundamentally change the pre-existing power distribution.

That said, fully-automated liberalism could help make global governance more liberal. Even permissioned blockchains, controlled by centralized actors, can empower traditionally underprivileged groups through the broadened set of opportunities for low-cost transnational interactions. For instance, blockchain-based remittance payments are cheaper than traditional payment systems. ${ }^{95}$ The related transaction cost savings generate real income growth that might empower marginalized actors. As described in the example of the climate DAO, marginalized actors will also be empowered through greencoin rewards for their local actions to further collective climate goals. As bearers of local knowledge, marginalized groups would also be empowered as they hold relevant information on which blockchain-based prediction markets depend. Yet, the possibility to earn greencoins is predisposed on aligned interests of states or other resourceful actors that establish such highpowered incentive schemes.

Permissionless blockchains even promise to directly empower individual citizens by disintermediating the roles of centralized actors and processes that traditionally underpin global governance. Blockchain may offer an entry point for weaker actors - specifically non-state actors - to challenge existing hierarchies, because it affords them a coordination device to organize decentralized action toward a collective goal without relying on recalcitrant governments. Such disintermediation, however, may not be normatively desirable if states are benevolent ${ }^{96}$; however, it would be desirable if states failed to be responsive to citizens, who then would need to rely on the blockchain as an alternative decentralized governance mechanism to affect global politics. Global carbon emissions trading market provides a case in point. Even without institutional support from governments, non-state actors would be able to trade carbon emissions through a blockchain-based governance system, although the system would be more effective with government support.

Despite some undeniable benefits in terms of empowering non-state actors, blockchain technology is unlikely to overcome existing hierarchies in the international system. In effect, it may even produce new kinds of power asymmetries.

\footnotetext{
${ }^{94}$ Needless to say, full automation is an ideal type here as there will always be some degree of human control retained, even if it is just the option of shutting down the internet.

${ }^{95}$ GSMA 2017, 25.

${ }^{96}$ Atzori 2015.
} 
Blockchain technology will challenge neither the primacy of states in global governance nor the preponderance of powerful states within the state community. If all states were to voluntarily participate in blockchain-based global governance, they would devise contracts with little obligation, as they must fear guaranteed punishment by smart contracts finding states to be violating their commitments. ${ }^{97}$ If the DAO only entailed contracts with hard obligations, (especially) powerful countries would not be willing to join the DAO, thus limiting its overall effectiveness. The promise of harder commitments that smart contracts can afford thus has the drawback of leaving those states out of its jurisdiction which would need to be constrained the most. The only way to incentivize participation by powerful states aside from collective mechanisms to allow them to renege on their commitments ${ }^{98}$ which are not easily achieved under crypto-law - is to unlock additional benefits for them, such as credible commitments for burden-sharing investments into the liberal order from non-state actors.

At the organization level - pertaining to the governance of blockchains - an increasing use of blockchain technology for global governance creates new power imbalances. The blockchain protocol is often hard-wired by developers, without much input from end-users. Knowledge to set up blockchain platforms is concentrated in the hands of a limited number of tech-savvy people, who can use it to their advantage on design issues - similar to how international bureaucrats can exploit their expertise for their own gain when designing new institutional progeny for member states. ${ }^{99}$ Hence, growing demand for blockchain-empowered systems to govern global challenges will likely empower a transnational elite of software developers at the expense of other societal groups. ${ }^{100}$

One might be tempted to argue that whether blockchain technology will alter the distribution of power in the international system crucially depends on their design. Permissionless blockchains promise to empower non-state actors by disintermediating central actors, although non-state actors can benefit even more from a hierarchically ordered governance system that intertwines these blockchains with permissioned blockchains that central actors would create to harness incentivized individual action within the framework of promoting international cooperation. For permissioned blockchains - typically controlled by central actors like states - the level of empowerment of non-state actors is likely to be limited. A unique focus on design for assessing power issues is limiting nonetheless because the design of blockchains occurs in the shadow of power and therefore will reflect existing hierarchies, with limited potential to make global politics more egalitarian. Considering the implications of blockchain technology for global order, it therefore seems that - as a new technology for decentralized governance - blockchain technology somewhat flattens hierarchies by allowing new actors to influence global politics more effectively than under current arrangements; however, states maintain the ability to control global governance by co-opting the technology to advance

\footnotetext{
${ }^{97}$ Downs et al. 1996.

${ }^{98}$ Stone 2011.

${ }^{99}$ Johnson and Urpelainen 2014.

${ }^{100}$ Campbell-Verduyn 2018, Narayanan et al. 2016, Ying-Hsieh et al. 2018.
} 
their own ends. Therefore, it seems that the rationalist view - expecting blockchain technology to be used by powerful actors to advance their predetermined interests is not fully convincing. Instead, in line with a constructivist view, governance by blockchain acts to reshape that activity and its meaning. ${ }^{101}$ This coincides with a co-evolutionary perspective on technological change, which argues that technology is not just formed by society but can also be active as structure, institution, or actor. $^{102}$

\section{Conclusion}

This paper probed whether and how blockchain technology could enhance global governance, with an illustration for climate governance. Although existing studies emphasize the apparent benefits of the technology related to information-sharing within traditional global governance structures, ${ }^{103}$ this paper sketched out the contours of a DAO comprising various sets of stakeholders interacting through smart contracts to achieve policy goals and to define the process by which future policy decisions will be made. Such a DAO would be implementable with available blockchain technology.

My theoretical analysis has shown that blockchain-enabled functionalities such as smart contracts could be deployed to promote cooperation in three ways: first, by leveraging collective information feeds, thereby helping resolve uncertainty about the state of world and state behaviors; second, to allay distribution problems in an efficient manner; and third, to allow states and other global governance actors to make more credible commitments to each other. Blockchain technology promises to offer new avenues for states to enlarge the set of circumstances under which they are willing to cooperate given ex ante preferences, for instance by leveraging new sources of information and by reducing downstream transaction costs that free up resources to allay distribution problems. Hence, even where scholars have traditionally thought that prospects of cooperation are grim - where states already live on the 'Pareto frontier" ${ }^{104}$ - a once-established blockchain-based global governance system with the aforementioned functionalities could enlarge the scope for cooperation.

My paper also interrogated whether blockchain-based global governance would be desirable. Although over-optimism that blockchain governance could instill liberal ideals into global politics must be dampened, there are undeniable benefits of blockchain-based governance compared to traditional global governance. The benefits of a blockchain-based governance system would be enhanced public accountability, through unrestricted read-only access to the blockchain, as well as increased democratic legitimacy, by including relevant stakeholders (such as NGOs) as participants in the consensus mechanism and by facilitating the transition from the current system in which power is distributed according to (largely non-transparent) lobbying contributions toward blockchain-based governance where parties stake

\footnotetext{
${ }^{101}$ Campbell-Verduyn 2018, 8.

${ }^{102}$ Dolata and Werle 2007.

${ }^{103}$ Chapron 2017, Rocamora and Amellina 2018, UNFCCC 2017.

${ }^{104}$ Krasner 1991.
} 
publicly for their interests. In addition, traditionally underprivileged stakeholders would likely be empowered as they hold important local information that would feed the oracles on which smart contracts depend to settle transactions. Blockchain technology also affords these stakeholders an opportunity to securely transact with each other, without relying on central authorities for this purpose. The costs of blockchain-based global governance are related to the well-known IR result that institutional design occurs in the shadow of power. States have incentives to establish some blockchain-based governance systems given that they can be designed not to threaten their own survival. ${ }^{105}$ This suggests the continued relevance of the liberal-institutionalist argument that a functional theory of cooperation is incomplete without consideration of political structures, specifically the distribution of power. It also suggests that blockchain-based global governance is unlikely to fulfill the normative ideal of 'fully-automated liberalism' in the shape under which it would ever come to fruition.

Although my case study considered climate policy, it might be useful to probe its generalizability to other issue areas. In many issue areas, blockchain-based governance would run counter to the interests of powerful actors, specifically influential states that would lose opportunities for power exercise if global affairs were governed by crypto-law. In some instances, smart contracts may also be collectively unprofitable because states would face high costs from the inflexibility that they imply - especially under conditions of uncertainty about the state of the world. Climate governance - given favorable conditions such as issue complexity and unmet potential for cooperation, resourceful domestic stakeholders with procooperation preferences, and a need for information systems that can validate climate-related events and policy measures - thus seems to be a most-likely case where a blockchain-based system is not completely out of question. Other issue areas with similar features and thus similar amenability to blockchain-based governance include trade, finance, and development.

Four issues merit particular attention in future research. First, who benefits and who loses from adopting blockchain-based global governance? A detailed answer to this question would provide further indication of how likely such adoption is under the given power distribution. The distributional consequences for states are not obvious, not least because they would hinge on the initial allocation of voting rights in blockchain-based governance systems. A once-established blockchain-based governance system would make the abuse of power more costly, whereas allowing for bottom-up governance by weaker actors, like developing countries and nongovernmental organizations, through smart contracts. A related issue is how blockchain-based governance affects principal-agent relationships between international organizations and member states. ${ }^{106}$ Blockchain technology could affect these relationships in multiple ways, depending on which functionalities are adopted. At the very least, international organizations would become more accountable to states to the extent that states would be able to more easily monitor

\footnotetext{
${ }^{105} \mathrm{~A}$ permissioned system is fully controlled by states, whereas a permissionless system circumvents them. Whether or not blockchains eliminate the nation-state depends on their designs.

${ }^{106}$ Conceição-Heldt 2017, Graham 2015, Manulak 2016, Nielson and Tierney 2003, Parízek 2016, Reinsberg 2019.
} 
the work of these organizations if they recorded their operations on the blockchain. At the extreme, under fully-automated liberalism, international organizations would be tasked primarily with validating external events and providing technical assistance, at the expense of fiduciary oversight roles and (possibly) policy coordination, which would be handled more efficiently through smart contracts. Clearly, this disintermediation potential of blockchains would raise opposition from international organizations.

A second issue - related to the first one - pertains to the governance of blockchain protocols. ${ }^{107}$ For example, whereas the Bitcoin network implies fully decentralized governance, the governance of the Bitcoin protocol is highly centralized controlled by its developers ${ }^{108}$ - and the Bitcoin consensus mechanism incentivizes the creation of centralized mining pools. The DAO represents a departure from centralized control over the blockchain protocol. Its internal governance rules can be altered through quasi-democratic consensus - with voting rights depending on the ownership of tokens. For some, the $2016 \mathrm{DAO}$ hack proved the failure of the DAO concept, ${ }^{109}$ whereas for others, the resorting to off-chain solutions represented a 'meta-governance structure' that was 'off-chain' but 'bound by the design of the technology'. ${ }^{110}$ Ultimately, the responsibility rests with actors collectively to decide through which mechanisms governance rules can be adapted. In this context, more research would be necessary to explore what kind of quorum is needed to simultaneously avoid control of the network by a cartel of powerful participants, whereas also preventing deadlock where a minority of nodes censor all new members.

A third area is challenges arising from the lack of interoperability with the offchain world. A key challenge is that many oracles are centralized, which makes them vulnerable as single points of failure whereas potentially undermining the veracity of data input to the blockchain. However, there are promising attempts to solve the 'oracle problem'. For example, Chainlink orchestrates a decentralized network of oracles relying on multiple sources of external information to evaluate queries from smart contracts in a trustworthy manner. ${ }^{111}$ Even if the oracle problem can be solved, there remains the question of how to constrain abusive uses of 'predictive analytics' afforded by the growing availability of 'big data' from the blockchain. This issue tends to be overlooked by scholarship hailing the ever-closer integration of blockchain with IoT devices. ${ }^{112}$ Although appropriate blockchain designs can likely mitigate this problem, more research is necessary.

A final area of inquiry would take up the challenge of developing metrics on the relative effectiveness of blockchain-based global governance against its present alternative. Such an analysis will remain impossible pending the actual use of blockchain and the generation of related data. If blockchain-based global governance

\footnotetext{
${ }^{107}$ Campbell-Verduyn and Hütten 2019.

${ }^{108}$ De Filippi and Loveluck 2016.

${ }^{109}$ DuPont 2018.

${ }^{110}$ Zwitter and Hazenberg 2020.

${ }^{111}$ Orcutt 2018. The Chainlink system supports many approaches of information aggregation from several oracles and has built-in features to incentivize truthful reporting and preventing coordinated malicious attacks (Ellis et al. 2017).

${ }^{112}$ Reyna et al. 2018.
} 
were to be implemented, the focus of inquiry would be to explain why some states participate in it whereas other states do not. In this regard, existing research in political economy - notably on the adoption of new technologies such as the internet ${ }^{113}$ - provides testable arguments. Although states cannot prevent individuals from using permissionless blockchains (unless they completely shut down the internet, which could then prompt individuals to use satellite technologies to connect to it), their participation in permissioned blockchains is voluntary. A straightforward prediction would be that demand for permissionless blockchains by non-state actors is higher in autocracies, whereas autocratic governments are less likely to join permissioned blockchains as their disutility of being exposed to transparency is greater. This exemplary discussion shows that the unique features of blockchain technology provide for new avenues of testing pertinent theories of IR and domestic politics.

Acknowledgements. I am particularly grateful to Christian Arnold, Malcolm Campbell-Verduyn, Giorgio Caselli, Simone Dietrich, Liane Hartnett, Robert Keohane, Andreas Kern, Axel Michaelowa, Cian O’Driscoll, Mette Eilstrup-Sangiovanni, Jason Sharman, Ian Shields, Ty Solomon, Tom Wilkinson, Joseph Zammit, Rene Reinsberg, Marek Olszewski, Sep Kamvar, the IT editors, and three anonymous reviewers for fruitful discussions and valuable comments.

Conflict of interest. The author declares no conflict of interest.

\section{References}

Abbott, Kenneth W., and Duncan Snidal. 1998. "Why States Act Through Formal International Organizations." Journal of Conflict Resolution 42 (1): 3-32.

Abbott, Kenneth W., and Duncan Snidal. 2000. "Hard and Soft Law in International Governance." International Organization 54 (3): 421-56.

Aganaba-Jeanty, Timiebi, Sam Anissimov, and Oonagh E. Fitzgerald. 2017. Blockchain ClimateCup Round Table. Toronto.

Andersen, Louise Riis. 2019. "Curb Your Enthusiasm: Middle-Power Liberal Internationalism and the Future of the United Nations.” International Journal 74 (1): 47-64.

Arruñada, Benito. 2018. "Impersonal Exchange." Minnesota Journal of Law, Science, and Technology 19: 55-105.

Atzori, Marcella. 2015. "Blockchain Technology and Decentralized Governance: Is the State Still Necessary?” SSRN Electronic Journal. http://www.ssrn.com/abstract=2709713. (Accessed 20 July 2018).

Baliga, Arati. 2017. "Understanding Blockchain Consensus Models." https://www.persistent.com/wp-content/uploads/2017/04/WP-Understanding-Blockchain-Consensus-Models.pdf.

Barber, Benjamin R. 2013. If Mayors Ruled the World: Dysfunctional Nations, Rising Cities. New Haven: Yale University Press.

Barnett, Michael N., and Martha Finnemore. 2004. Rules for the World: International Organizations in Global Politics. Ithaca: Cornell University Press.

Baumann, Tom. 2018. "Using Blockchain to Achieve Climate Policy Outcomes." IETA Insights Policy Brief. Beckert, Jens. 1999. "Agency, Entrepreneurs, and Institutional Change. The Role of Strategic Choice and Institutionalized Practices in Organizations.” Organization Studies 20 (5): 777-99.

Bendrath, Ralf, and Milton Mueller. 2011. "The End of the Net as We Know It? Deep Packet Inspection and Internet Governance." New Media and Society 13 (7): 1142-60.

Boylan, David. 2001. Defusing Democracy: Central Bank Independence and the Transition from Authoritarian Rule. Ann Arbor: University of Michigan Press.

Brown, Chris, Terry Nardin, and Nicholas Rengger. 2002. International Relations in Political Thought: Texts from the Ancient Greeks to the First World War. New York: Cambridge University Press.

\footnotetext{
${ }^{113}$ Milner 2006.
} 
Buerger, Hans-Helge. 2016. "Ethereum White Paper: A Next-Generation Smart Contract and Decentralized Application Platform." https://github.com/ethereum/wiki/wiki/White-Paper.

Bulkeley, Harriet et al. 2014. Transnational Climate Change Governance. Cambridge: Cambridge University Press.

Buterin, Vitalik. 2014. A next-Generation Smart Contract and Decentralized Application Platform.

Campbell-Verduyn, Malcolm. 2018. Bitcoin and Beyond: Cryptocurrencies, Blockchains, and Global Governance. London: Routledge.

Campbell-Verduyn, Malcolm, and Marcel Goguen. 2018. "The Mutual Constitution of Technology and Global Governance: Bitcoin, Blockchains, and the International Anti-Money-Laundering Regime." In Bitcoin and Beyond: Cryptocurrencies, Blockchains, and Global Governance, edited by Malcolm Campbell-Verduyn, 69-87. London: Routledge.

Campbell-Verduyn, Malcolm, Marcel Goguen, and Tony Porter. 2019. "Finding Fault Lines in Long Chains of Financial Information." Review of International Political Economy 26 (5): 911-37.

Campbell-Verduyn, Malcolm, and Moritz Hütten. 2019. "Anticipating Decentralization Through Protocological Control: International Organizations and the Standardization of Blockchain Technology within Financial/Security Infrastructures.” University of Groningen.

Chapron, Guillaume. 2017. "The Environment Needs Cryptogovernance." Nature 545 (7655): 403-5.

Conceição-Heldt, Eugénia da. 2017. "Regaining Control of Errant Agents? Agency Slack at the European Commission and the World Health Organization." Cooperation and Conflict 52 (4): 469-84.

Dacin, Tina M., Jerry Goodstein, and Richard W. Scott. 2002. "Institutional Theory and Institutional Change: Introduction to the Special Research Forum." The Academy of Management Journal 45 (1): 43-56.

Dai, Xinyuan. 2007. International Institutions and National Policies. New York: Cambridge University Press.

De Filippi, Primavera, and Benjamin Loveluck. 2016. "The Invisible Politics of Bitcoin: Governance Crisis of a Decentralized Infrastructure." Internet Policy Review 5 (4): 1-32.

De Filippi, Primavera, and Samer Hassan. 2016. "Blockchain technology as a regulatory technology: From code is law to law is code." First Monday, 21(12): https://doi.org/10.5210/fm.v21i12.7113.

DeNardis, Laura. 2014. The Global War for Internet Governance. New Haven: Yale University Press.

Diedrich, Henning. 2016. Ethereum: Blockchains, Digital Assets, Smart Contracts, Decentralized Autonomous Organizations. London: Wildfire Publishing.

Dietrich, Simone, and Amanda Murdie. 2017. "Human Rights Shaming Through INGOs and Foreign Aid Delivery." Review of International Organizations 12 (1): 95-120.

Dobbin, Frank, Beth A. Simmons, and Geoffrey Garrett. 2007. "The Global Diffusion of Public Policies: Social Construction, Coercion, Competition, or Learning?" Annual Review of Sociology 33 (1): 449-72.

Dolata, Ulrich, and Raymund Werle. 2007. "Bringing Technology Back in': Technik Als Einflussfaktor Sozioökonomischen Und Institutionellen Wandels." In Gesellschaft Und Die Macht Der Technik: Sozioökonomischer Und Institutioneller Wandel Durch Technisierung, edited by Ulrich Dolata, and Raymund Werle, 15-43. Frankfurt: Campus.

Downs, George W., David M. Rocke, and Peter N. Barsoom. 1996. "Is the Good News about Compliance Good News about Cooperation?" International Organization 50 (3): 379-406.

DuPont, Quinn. 2018. "Experiments in Algorithmic Governance: A History and Ethnography of 'The DAO,' a Failed Decentralized Autonomous Organization.” In Bitcoin and Beyond: Cryptocurrencies, Blockchains, and Global Governance, edited by Malcolm Campbell-Verduyn, 157-77. London: Routledge.

Ellis, Steve, Ari, Juels, and Sergey, Nazarov. 2017. "ChainLink: A Decentralized Oracle Network." White Paper v1.0. https://crushcrypto.com/wp-content/uploads/2017/09/LINK-Whitepaper.pdf (accessed 1 January 2020).

Falkner, Robert. 2016. "The Paris Agreement and the New Logic of International Climate Politics." International Affairs 92 (5): 1107-25.

Fearon, James D. 1995. "Rationalist Explanations for War." International Organization 49 (3): 379-414.

Fu, Bailu, Zhan Shu, and Xiaogang Liu. 2018. "Blockchain Enhanced Emission Trading Framework in Fashion Apparel Manufacturing Industry." Sustainability 10 (4): 1-19.

Graham, Erin R. 2015. Money and Multilateralism: How Funding Rules Constitute IO Governance. International Theory 7 (1): 162-94. 
GSMA. 2017. Blockchain for Development: Emerging Opportunities for Mobile, Identity and Aid. London. https://www.gsma.com/mobilefordevelopment/wp-content/uploads/2017/12/Blockchain-forDevelopment.pdf.

Haddock, Bruce. 1999. "State and Nation in Mazzini’s Political Thought." History of Political Thought 20 (2): $313-36$.

Hale, Thomas. 2016. "'All Hands on Deck': The Paris Agreement and Nonstate Climate Action." Global Environmental Politics 16 (3): 12-22.

Hart, Oliver, and John Moore. 1999. "Foundations of Incomplete Contracts." Review of Economic Studies 66 (1): 115-38.

Heeks, Richard, and Savita Bailur. 2007. "Analyzing E-Government Research: Perspectives, Philosophies, Theories, Methods, and Practice." Government Information Quarterly 24 (2): 243-65.

Hooghe, Liesbet, Tobias Lenz, and Gary Marks. 2018. "Contested World Order: The Delegitimation of International Governance." Review of International Organizations 14 (4): 731-43.

Hütten, Moritz, and Matthias Thiemann. 2018. "Moneys at the Margins: From Political Experiment to Cashless Societies." In Bitcoin and Beyond: Cryptocurrencies, Blockchains, and Global Governance, edited by Malcolm Campbell-Verduyn, 25-47. London: Routledge.

Ikenberry, John G. 2001. After Victory: Institutions, Strategic Restraint, and the Rebuilding of Order after Major Wars. Princeton, NJ: Princeton University Press.

Ikenberry, John G. 2018. “The End of Liberal International Order?” International Affairs 94 (1): 7-23.

Jasanoff, Sheila. 2004. States of Knowledge: The Co-Production of Science and the Social Order. London: Routledge.

Jervis, Robert. 1999. "Realism, Neoliberalism, and Cooperation: Understanding the Debate." International Security 24 (1): 42-63.

Johnson, Tana, and Andrew Heiss. 2018. "Liberal Institutionalism." In International Organization and Global Governance, edited by. Thomas G Weiss and Rorden Wilkinson, 123-34. London: Routledge.

Johnson, Tana, and Johannes Urpelainen. 2014. "International Bureaucrats and the Formation of Intergovernmental Organizations: Institutional Design Discretion Sweetens the Pot.” International Organization 68 (1): 177-209.

Just, Natascha, and Michael Latzer. 2017. "Governance by Algorithms: Reality Construction by Algorithmic Selection on the Internet." Media, Culture and Society 39 (2): 238-58.

Kelley, Judith G., and Beth A. Simmons. 2015. "Politics by Number: Indicators as Social Pressure in International Relations." American Journal of Political Science 59 (1): 55-70.

Keohane, Robert O. 1984. After Hegemony: Cooperation and Discord in the World Political Economy. Princeton, NJ: Princeton University Press.

Keohane, Robert O., and Lisa L. Martin. 1995. "The Promise of Institutionalist Theory.” International Security 20 (1): 39-51.

Kern, Andreas, Bernhard Reinsberg, and Matthias Rau-Göhring. 2019. IMF conditionality and Central Bank Independence. European Journal of Political Economy 59: 212-29.

Koremenos, Barbara, Charles Lipson, and Duncan Snidal. 2001. "The Rational Design of International Institutions." International Organization 55 (4): 761-99.

Krasner, Stephen D. 1991. "Global Communications and National Power: Life on the Pareto Frontier." World Politics 43 (April): 336-66.

Krisch, Nico. 2017. "Liquid Authority in Global Governance." International Theory 9 (2): 237-60.

Krishna, Arvind, Martin Fleming, and Solomon Assefa. 2017. "Instilling Digital Trust." In Digital Revolutions in Public Finance, edited by. Sanjeev Gupta, Michael Keen, Alpa Shah, and Geneviève Verdier, 173-97. Washington, DC: International Monetary Fund.

Lessig, Lawrence. 1999. Code and Other Laws of Cyberspace. New York: Basic Books.

Lundgren, Magnus. 2016. "Which Type of International Organizations Can Settle Civil Wars?" The Review of International Organizations 12 (4): 613-41.

Manulak, Michael W. 2016. "Leading by Design: Informal Influence and International Secretariats." Review of International Organizations 12 (4): 497-522.

Martin, Lisa L. 2017. "International Institutions: Weak Commitments and Costly Signals." International Theory 9 (3): 353-80.

Mattern, Janice Bially, and Ayșe Zarakol. 2016. "Hierarchies in World Politics.” International Organization 70 (3): 623-54. 
Milner, Helen V. 2006. “The Digital Divide: The Role of Political Institutions in Technology Diffusion.” Comparative Political Studies 39 (2): 176-99.

Morrow, James D. 1994. "International Organization Foundation Modeling the Forms of International Cooperation: Distribution versus Information.” International Organization 48 (3): 387-423.

Musgrave, Paul, and Daniel H Nexon. 2018. "Defending Hierarchy from the Moon to the Indian Ocean: Symbolic Capital and Political Dominance in Early Modern China and the Cold War." International Organization, 72 (3), 591-62.

Nakamoto, Satoshi. 2008. "Bitcoin: A Peer-to-Peer Electronic Cash System." https://bitcoin.org/bitcoin.pdf.

Narayanan, A et al. 2016. Bitcoin and Cryptocurrency Technologies: A Comprehensive Introduction. Princeton, NJ: Princeton University Press.

Nielson, Daniel L., and Michael J. Tierney. 2003. "Delegation to International Organizations: Agency Theory and World Bank Environmental Reform." International Organization 57 (2): 241-76.

North, Douglass C. 1990. Institutions, Institutional Change and Economic Performance. New York: Cambridge University Press.

Orcutt, Mike. 2018. Blockchain smart contracts are finally good for something in the real world. MIT Technology Review (19 November 2018).

Parízek, Michal. 2016. "Control, Soft Information, and the Politics of International Organizations Staffing." Review of International Organizations 12 (4): 559-83.

Pisa, Michael. 2018. Reassessing Expectations for Blockchain and Development. Washington, DC: USAID.

Reinsberg, Bernhard. 2019. "Blockchain technology and the governance of foreign aid." Journal of Institutional Economics 15 (3): 413-29.

Reyna, Ana, Cristian Martín, Jaime Chen, Enrique Soler, and Manuel Díaz. 2018. "On Blockchain and its Integration with IoT. Challenges and Opportunities.” Future Generation Computer Systems 88: 173-90.

Rocamora, Alexis, and Aryanie Amellina. 2018. Blockchain Applications and the Sustainable Development Goals: Analysis of Blockchain Technology's Potential in Creating a Sustainable Future. Kamiyamaguchi.

Rosenau, James N. 1995. "Governance in the Twenty-First Century." Global Governance 1 (1): 1-15.

Rosendorff, Peter B., and Helen V. Milner. 2001. “The Optimal Design of International Trade Institutions: Uncertainty and Escape Clauses.” International Organization 55 (4): 829-57.

Ruggie, John Gerard. 1975. "International Responses to Technology: Concepts and Trends." International Organization 29 (3): 557-83.

Simmons, Beth A. 2010. "Treaty Compliance and Violation.” Annual Review of Political Science 13 (1): $273-96$. Simmons, Beth A., and Allison Danner. 2010. "Credible Commitments and the International Criminal Court.” International Organization 64 (2): 225-56.

Simmons, Beth A., and Z. Elkins. 2004. "The Globalization of Liberalization: Policy Diffusion in the International Political Economy.” American Political Science Review 98 (1): 171-90.

Sklaroff, Jeremy M. 2017. "Smart Contracts and the Cost of Inflexibility." University of Pennsylvania Journal of International Law 166: 263-303.

Slaughter, Anne-Marie. 2016. "How to Succeed in the Networked World: A Grand Strategy for the Digital Age.” Foreign Affairs 95: 76-89.

Stein, Arthur A. 1982. "Coordination and Collaboration: Regimes in an Anarchic World.” International Organization 36 (2): 299-324.

Stein, Arthur A. 2009. "Neoliberal Institutionalism." In The Oxford Handbook of International Relations, edited by Christian Reus-Smit and Duncan Snidal, 1-21. Oxford: Oxford University Press.

Stone, Randall W. 2011. Controlling Institutions: International Organizations and the Global Economy. New York: Cambridge University Press.

Swan, Melanie. 2015. Blockchain: Blueprint for a New Economy. Sebastopol, CA: O’Reilly Media, Inc.

Szabo, Nick. 1994. Smart Contracts.

Tallberg, Jonas, Thomas Sommerer, and Theresa Squatrito. 2016. "Democratic Memberships in International Organizations: Sources of Institutional Design.” Review of International Organizations 11 (1): 59-87.

Tapscott, Don, and Alex Tapscott. 2016. Blockchain Revolution: How the Technology Behind Bitcoin Is Changing Money, Business, and the World. New York: Penguin Random House.

Tapscott, Don, and Alex Tapscott. 2017. "Realizing the Potential of Blockchain a Multistakeholder Approach to the Stewardship of Blockchain and Cryptocurrencies.” Available from www.weforum.org. 
Troyjo, Marcos, Julia Quintella, and Fracisco Da Silva. 2017. Building ITTI: Intelligent Tech \& Trade Initiative. Sao Paolo. http://itti-global.org/2017/12/.

Truby, Jon. 2018. "Decarbonizing Bitcoin: Law and Policy Choices for Reducing the Energy Consumption of Blockchain Technologies and Digital Currencies." Energy Research \& Social Science, 44: 399-410.

UNFCCC. 2017. "How Blockchain Technology Could Boost Climate Action." https://unfccc.int/news/howblockchain-technology-could-boost-climate-action (30 March 2018).

Valkenburgh, Peter Van. 2016. Open Matters: Why Permissionless Blockchains are Essential to the Future of the Internet. CoinDesk.

Vaubel, Roland, Axel Dreher, and Ugurlu Soylu. 2007. "Staff Growth in International Organizations: A Principal-Agent Problem? An Empirical Analysis.” Public Choice 133 (3-4): 275-95.

Voeten, Erik. 2014. "Does Participation in International Organizations Increase Cooperation?" Review of International Organizations 9 (3): 285-308.

von Stein, Jana. 2005. "Do Treaties Constrain or Screen? Selection Bias and Treaty Compliance." American Political Science Review 99 (4): 611-22.

Wigley, Bob, and Nicolas Cary. 2017. The Future is Decentralised: Blockchains, Distributed Ledgers, and the Future of Sustainable Development.

Wilson, Woodrow. 2006. Essential Writings and Speeches of the Scholar-President. New York: NYU Press.

Wood, Gavin. 2017. Polkadot: Vision for a Heterogeneous Multi-Chain Framework. https://github.com/ w3f/polkadot-white-paper/raw/master/PolkaDotPaper.pdf.

Wright, Aaron, and Primavera De Filippi. 2015. Decentralized Blockchain Technology and the Rise of Lex Cryptographia.

Ying-Hsieh, Ying, Jean-Philippe Vergne, and Sha Wang. 2018. "The Internal and External Governance of Blockchain-Based Organizations: Evidence from Cryptocurrencies." In Bitcoin and Beyond: Cryptocurrencies, Blockchains, and Global Governance, edited by Malcolm Campbell-Verduyn, 48-68. London: Routledge.

Zwitter, Andrej. 2015. "Big Data and International Relations." Ethics and International Affairs 29 (4): 37789.

Zwitter, Andrej, and Jilles Hazenberg. 2020. "Decentralized Network Governance: Blockchain Technology and the Future of Regulation." Frontiers in Blockchain 3: 1-12.

Zysman, John, and Abraham L. Newman. 2006. How Revolutionary Was the Digital Revolution?: National Responses, Market Transitions, and Global Technology. Stanford: Stanford University Press.

Cite this article: Reinsberg, B. 2021. "Fully-automated liberalism? Blockchain technology and international cooperation in an anarchic world." International Theory 13, 287-313, doi:10.1017/S1752971920000305 